\title{
Gas Flow Rate and Scanning Speed influence on microstructure and Microhardness Property of Laser Metal Deposited Titanium-alloy
}

\author{
Rasheedat M. Mahamood ${ }^{1, a^{*}}$ and Esther T. Akinlabi ${ }^{1}$ \\ ${ }^{1}$ Department of Mechanical Engineering Science, University of Johannesburg, \\ Auckland Park Campus, Johannesburg, South Africa, 2006. \\ amahamoodmr2009@gmail.com
}

Keywords: Additive manufacturing, Gas flow rate, Laser metal deposition, Microhardness, Scanning speed, Titanium alloy.

\begin{abstract}
Laser metal deposition process is an additive manufacturing technology that is capable of producing three dimensional components as well as repair of an existing worn out components. Processing parameters play an important role on the resulting properties of the processed materials using the laser metal deposition process. This study investigates the influence of gas flow rate and scanning speed on the microstructural and microhardness properties of laser metal deposited Ti6Al4V, an important titanium alloy used in the aerospace industries. Nd-YAG laser was used in this work with the maximum laser power of $4.0 \mathrm{~kW}$. The laser power used was $3.0 \mathrm{~kW}$ and the powder flow rate of $2.88 \mathrm{~g} / \mathrm{min}$ was maintained throughout the experiments. The scanning speed was set between 0.01 and $0.04 \mathrm{~m} / \mathrm{s}$ while the gas flow rate was varied between 1 and $41 / \mathrm{min}$. The microstructures of the samples were studied using optical microscope while the microhardness profiling was conducted using microhardness indenter. The results showed that, as the scanning speed was increased, the microstructure changed from fine basketweave structure to coarse martensitic structure. The microhardness was found to increase with increasing scanning speed. By increasing the gas flow rate results in decrease in microhardness values while the microstructure was observed to change from martensitic structure to basketweave structure. The result from this study is especially useful in repair application in order to achieve the desired properties.
\end{abstract}

\section{Introduction}

Ti6Al4V is an important aerospace alloy and the most widely used titanium alloy, because of the excellent properties it possess which include high strength to weight ratio and excellent corrosion resistance properties $[1,2]$. Though Ti6Al4V has excellent properties but it is classified as difficult to machine material [3]. The use of traditional manufacturing method to process Ti6Al4V challenging and time consuming. Laser metal deposition process that belongs to the directed energy deposition class of additive manufacturing technology is a candidate alternative manufacturing process that can be used to process difficult to machine materials such as Ti6Al4V because it is a contactless manufacturing process and it does not come in contact with the work material. Laser metal deposition process can also be used to repair existing worn out part as well as in the fabrication of functionally graded materials [4$6]$.

Laser metal deposition (LMD) process is a fairly new additive manufacturing process that has gain a lot of interest in the research community because of its importance and the promises it offers. Aside from the ability of the LMD process in the fabrication of near net shape part that is made directly from the three dimensional (3D) computer aided design (CAD) image of the part through the materials addition layer by layer, LMD is also capable of repairing high valued parts that were not repairable in the past [7-9]. It also promised to reduce buy-to-fly ratio in the aerospace industry, ability to produce composite and functionally graded materials and its mass customization capability [10-13]. 


\section{Experimental Method}

The materials used in this study are the Ti6Al4V powder and Ti6Al4V substrate. The Ti6Al4V powder used is $99.6 \%$ pure and it is of particle size range between 150 and $250 \mu \mathrm{m}$. The Ti6Al4V substrate used is also a $99.6 \%$ pure square sheet of $5 \mathrm{~mm}$. Nd-YAG laser with maximum capacity of 4 $\mathrm{kW}$ was used for the LMD process. The laser was attached to the end effector of a Kuka robot with the coaxial powder nozzle. The experimental set-up used in this study is available at the CSIR, National Laser Center Pretoria, South Africa. An improvised glove box was provided to prevent the deposited samples from environmental such as atmospheric oxygen and Nitrogen. The laser metal deposition process was achieved by the laser beam that is used to create a melt-pool on the surface of the substrate while the powder material is then delivered into the melt-pool. A solid track of the deposited sample is seen on the path of the laser beam when the melt-pool solidifies. The experimental set-up and the schematic diagram of the LMD process are shown in Fig. 1a and Fig. 1b respectively. The laser power and the powder flow rate used in this study were fixed at $3.0 \mathrm{~kW}$ and $2.88 \mathrm{~g} / \mathrm{min}$ respectively. The scanning speed was varied between 0.01 and $0.04 \mathrm{~m} / \mathrm{s}$ while the gas flow rate was varied between 1 and $4 \mathrm{l} / \mathrm{min}$. The processing parameters used are presented in Table 1 . The laser beam spot size was maintained at $2 \mathrm{~mm}$ through the constant focal length of $195 \mathrm{~mm}$ above the substrate. The substrate was sandblasted and washed with acetone to prepare the surface to aid the laser energy absorption prior to the deposition process.

A total of eight (8) samples were produced according to the processing parameters in Table 1. After the experiments were completed, the samples were cut in transverse direction to reveal the cross section of the samples. The cut samples were mounted in resin, ground and polished following the standard metallurgical preparation of titanium and its alloys [14]. The polished samples were then etched using the Kroll's reagent. The microstructures of the samples were observed under the optical microscopy (OP) by Olympus BX51M.

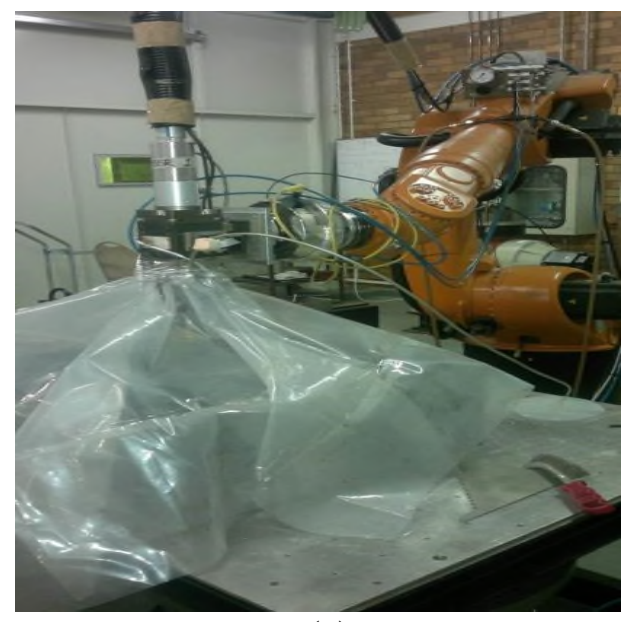

(a)

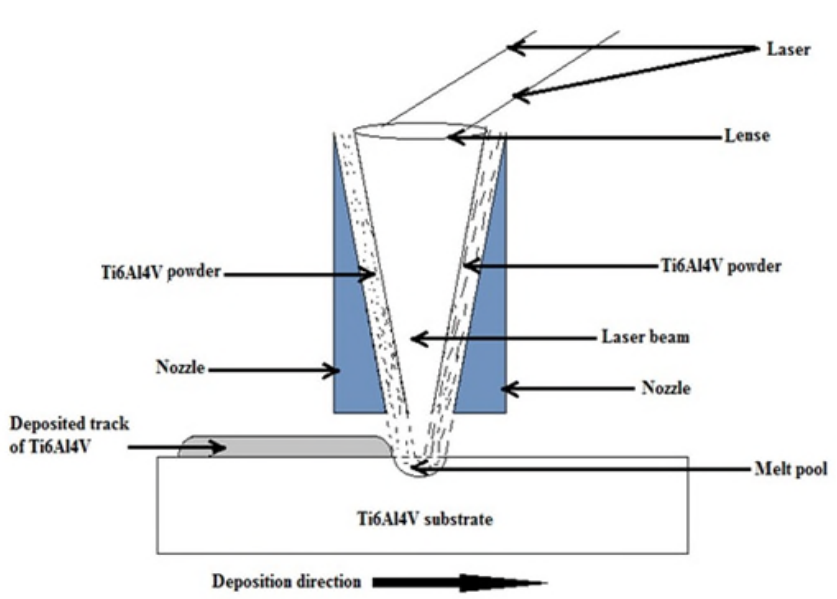

(b)

Fig. 1 (a) Pictorial diagram of the experimental set-up

(b) Schematic diagram of the LMD process (adapted from Mahamood et al. [15]).

The microhardness of the polished samples were measured using the Metkon microhardness tester with a load of $500 \mathrm{~g}$ and a dwelling time of $15 \mathrm{~s}$. The distance between indentation were kept at $15 \mu \mathrm{m}$. The microhardness was measured according to the ASTM standard [16]. 
Table 1. Experimental Matrix.

\begin{tabular}{|c|c|c|c|c|}
\hline $\mathrm{S} / \mathrm{N}$ & $\begin{array}{c}\text { Scanning Speed } \\
(\mathrm{m} / \mathrm{s})\end{array}$ & $\begin{array}{c}\text { Gas Flow Rate } \\
(1 / \mathrm{min})\end{array}$ & Laser Power $(\mathrm{kW})$ & $\begin{array}{c}\text { Powder Flow Rate } \\
(\mathrm{g} / \mathrm{min})\end{array}$ \\
\hline 1 & 0.01 & 4 & 3.0 & 2.88 \\
\hline 2 & 0.02 & 4 & 3.0 & 2.88 \\
\hline 3 & 0.03 & 4 & 3.0 & 2.88 \\
\hline 4 & 0.04 & 4 & 3.0 & 2.88 \\
\hline 5 & 0.04 & 3 & 3.0 & 2.88 \\
\hline 6 & 0.04 & 2 & 3.0 & 2.88 \\
\hline 7 & 0.04 & 1 & 3.0 & 2.88 \\
\hline
\end{tabular}

\section{Results and Discussions}

The microhardness results are presented in Table 2. The graph of the microhardness against the scanning speed is shown in Fig. 2. The microstructure of the Ti6Al4V substrate and the morphology of the Ti6Al4V powder are shown in Fig. 3a and Fig. 3b respectively. The microstructure of the Ti6Al4V substrate is characterized by alpha (bright coloured) and beta phase (dark coloured) grains. The microstructure of the sample at scanning speed of $0.01 \mathrm{~m} / \mathrm{s}$ is shown in Fig. 4a while the micrograph of the sample at a scanning speed of $0.04 \mathrm{~m} / \mathrm{s}$ is shown in Fig. 4b. The microhardness against the powder flow rate is shown in Fig. 5. The micrograph of the sample at powder flow rate of $11 / \mathrm{min}$ and that of the sample at $31 / \mathrm{min}$ are shown in Fig. $6 \mathrm{a}$ and Fig. $6 \mathrm{~b}$ respectively.

Table 2. Results for the Average Microhardness

\begin{tabular}{|c|c|c|c|}
\hline $\mathrm{S} / \mathrm{N}$ & Scanning Speed $(\mathrm{m} / \mathrm{s})$ & Gas Flow Rate $(1 / \mathrm{min})$ & $\begin{array}{c}\text { Average } \\
\text { Microhardness (HV) }\end{array}$ \\
\hline 1 & 0.01 & 4 & 320.18 \\
\hline 2 & 0.02 & 4 & 335.01 \\
\hline 3 & 0.03 & 4 & 352.05 \\
\hline 4 & 0.04 & 4 & 371.45 \\
\hline 5 & 0.04 & 3 & 374.13 \\
\hline 6 & 0.04 & 2 & 377.07 \\
\hline 7 & 0.04 & 1 & 378.81 \\
\hline Substrate & - & - & 300.00 \\
\hline
\end{tabular}

The microhardness is found to increase with an increase in scanning speed. The reason for this is that, at low scanning speed, the laser material interaction time is high and resulted in a larger melt pool. The solidification of larger melt pool takes longer which resulted in the formation of basketwoven (Widmanstätten) microstructure as shown in Fig. 4a. At higher scanning speed, the laser material interaction time is shorter which resulted in the formation of martensitic microstructure (see Fig. 4b) due to rapid solidification of smaller melt pool formed at high scanning speed. 


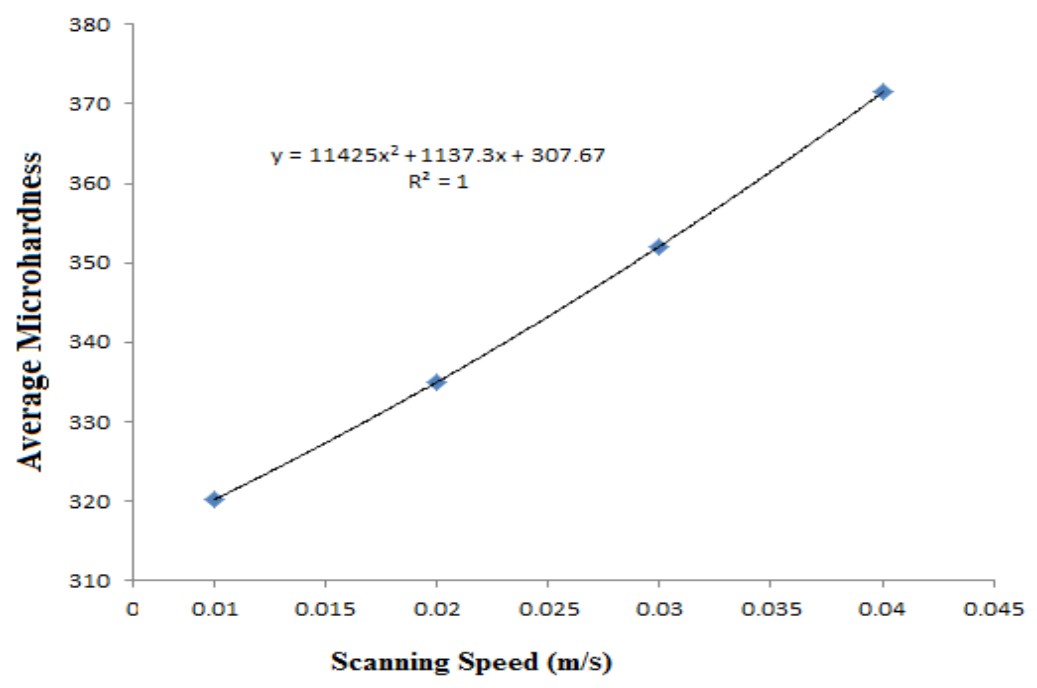

Fig. 2 Graph of microhardness versus scanning speed.

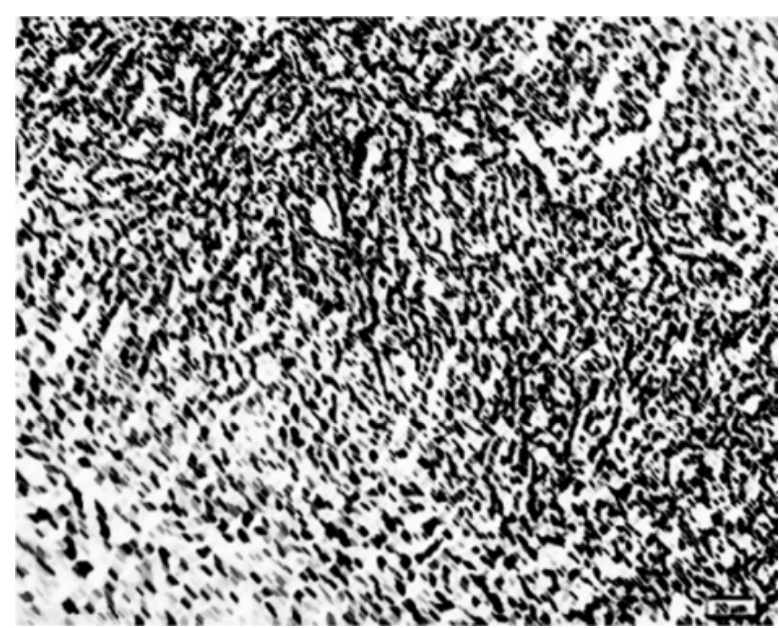

(a)

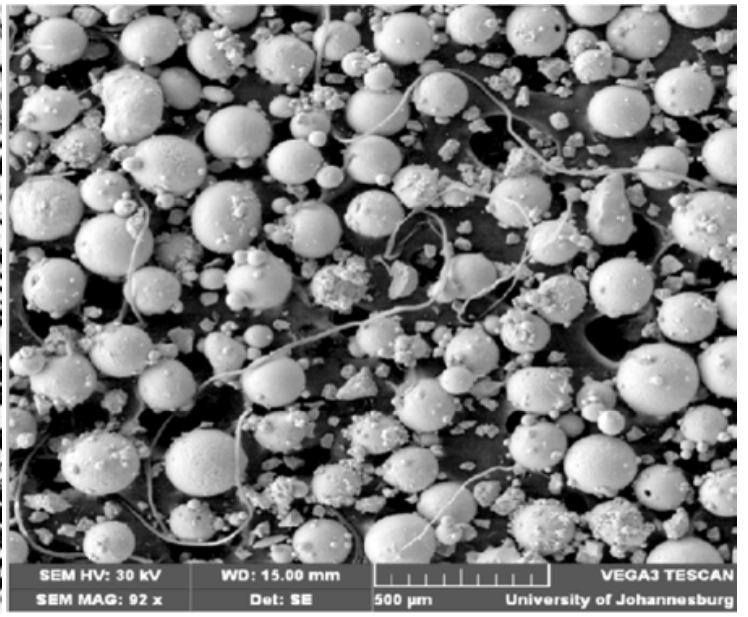

(b)

Fig. 3 (a) Micrograph of the surface of the Ti6A14V substrate (b) SEM micrograph of the Ti6Al4V powder [17].

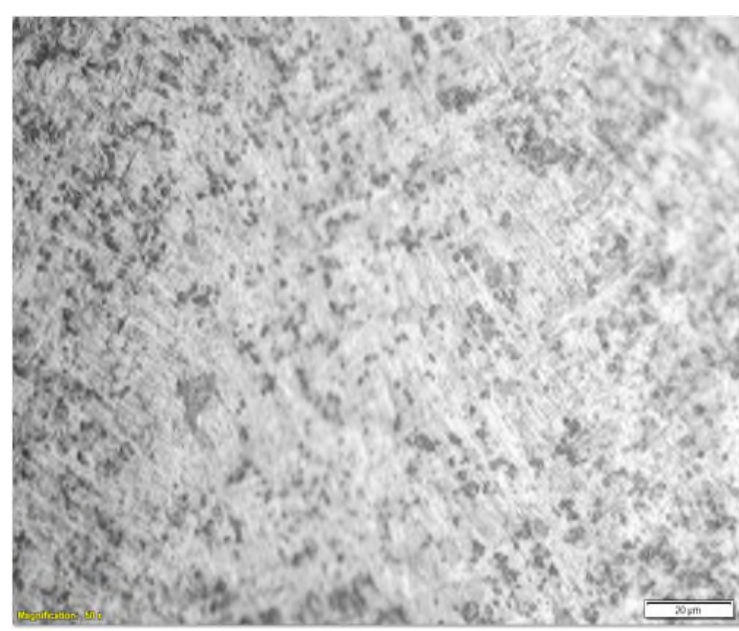

(a)

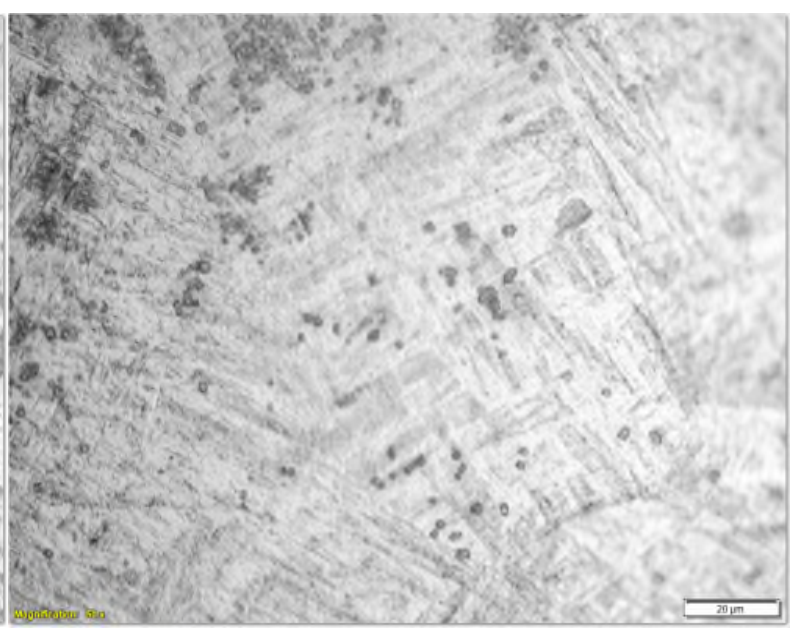

(b)

Fig. 4 Micrograph of sample at scanning speed of (a) $0.01 \mathrm{~m} / \mathrm{s}$ (b) $0.04 \mathrm{~m} / \mathrm{s}$. 
The microhardness was found to reduce with increased gas flow rate as shown in Fig. 5. The low microhardness at high gas flow rate could be as a result of higher agitation of the deposited powder by the high gas flow rate that resulted in more proper melting of the powder resulting in larger melt pool. At lower scanning speed, there is less steering action of the gas and the melt pool form is smaller that resulted in rapid solidification and hence formation of martensitic grains as shown in Fig. 6a. The slower solidification of large melt pool resulted in the formation of Widmanstätten microstructure as shown in Fig. 6b.

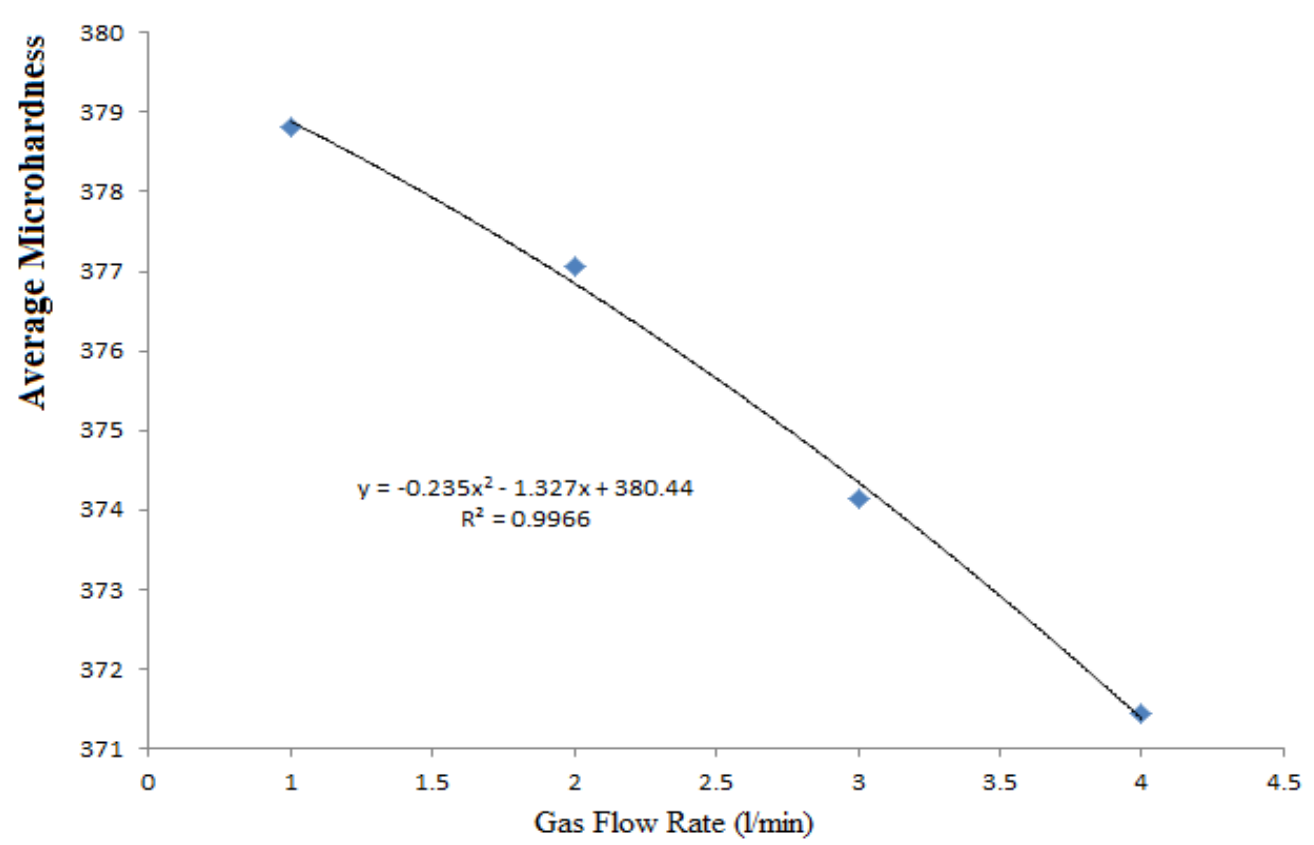

Fig. 5 Graph of microhardness against the gas flow rate.

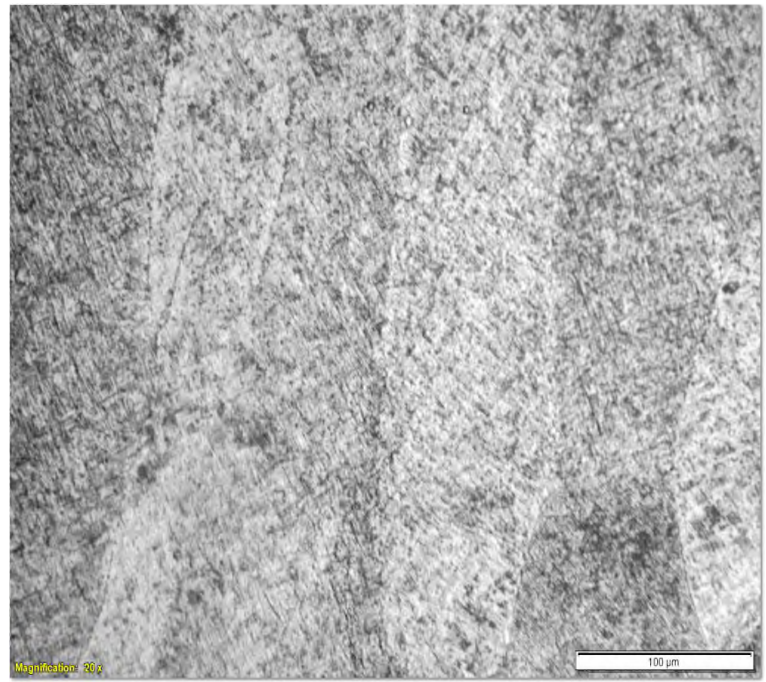

(a)

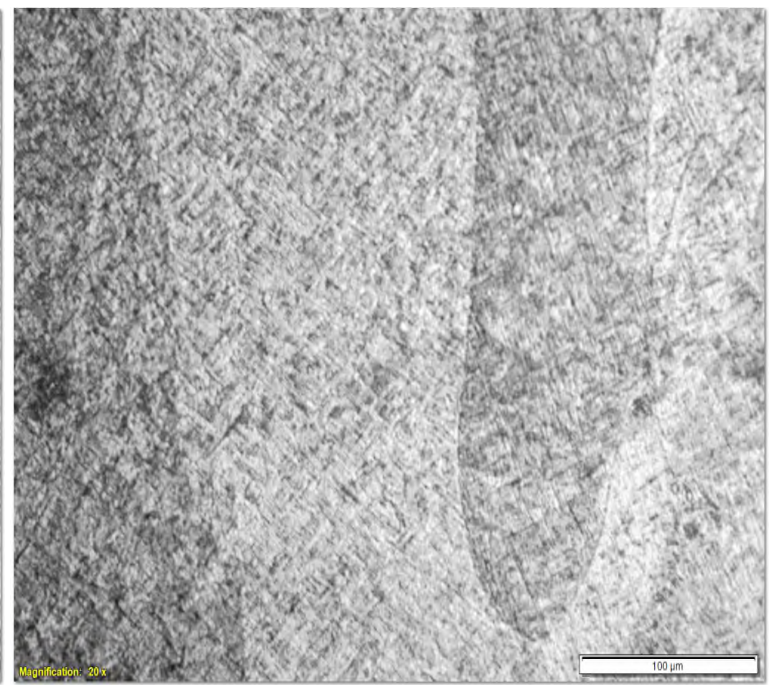

(b)

Fig. 6 Micrograph of sample at powder flow rate of (a) $11 / \mathrm{min}$ (b) $3 \mathrm{l} / \mathrm{min}$ ). 


\section{Conclusion}

The influence of scanning speed and gas flow rate on the microstructure and microhardness was investigated in this study. The laser power and powder flow rate were fixed while the scanning speed and the gas flow rate were varied. The microhardness was found to increase with an increase in scanning speed while it decreases with an increase in gas flow rate. The rate at which the microhardness changes with change in scanning speed is higher than the rate it changed with change in gas flow rate. This showed that the scanning speed is more significant than gas flow rate. This study showed that the processing parameters should be adequately controlled in laser metal deposition process to achieve the desired properties. This study is useful in repair of high valued components using the laser metal deposition process.

\section{Acknowledgement}

This work was supported by University of Johannesburg research council and Rental Pool Grant of the National Laser Centre - Council of Scientific and Industrial Research (NLC-CSIR), Pretoria South Africa

\section{References}

[1] G. Lütjering, J. C. Williams, Titanium, Springer, Berlin, Germany, (2003).

[2] Y. Lu, H. B. Tang, Y. L. Fang, D. Liu, H. M. Wang, Microstructure evolution of sub-critical annealed laser deposited Ti-6Al-4V alloy, Mater. Des. 37 (2012) 56-63.

[3] Z. M, Wang, E. O. Ezugwu, Titanium Alloys and Their Machinability a Review. J. Mater. Proc. Tech. 68 (1997) 262-270.

[4] B. Graf, A. Gumenyuk, M. Rethmeier, Laser metal deposition as repair technology for stainless steel and Titanium alloys. Phys. Proc. 39 (2012) 376-381.

[5] A. J. Pinkerton, W. Wang, L. Li, Component repair using laser direct metal deposition, J. Eng. Manuf. 222 (2008) 827-836.

[6] R. M. Mahamood, E. T. Akinlabi, M. Shukla, S. Pityana. Functionally graded material: An overview. Proceedings of the World Congress on Engineering, III, WCE 2012, July 4-6, 2012, London, U.K. (2012), pp. 1593-1597.

[7] M. Peters, J. Kumpfert, C.H. Ward, C. Leyens, Titanium alloys for aerospace applications, in: Titanium and Titanium alloys, Adv. Eng. Mater. 5 (2003) 419-427.

[8] A. G. Ermachenko, R. Ya. Lutfullin, R. R. Mulyukov, Advanced technologies of processing titanium alloys and their applications in industry, Rev. Adv. Mater. Sci. 29 (2011) 68-82.

[9] M. J. Donachi, Titanium-A technical guide, 2nd ed.Metals Park, OH: ASM International, (2000).

[10] I. Gibson, B. Stucker, D. W. Rosen, Additive manufacturing technologies: Rapid prototyping to direct digital manufacturing, Springer, (2009).

[11] E. Brandl, V. Michailov, B. Viehweger, C. Leyens, Deposition of Ti-6Al-4V using laser and wire, part I: Microstructural properties of single beads, Surf. Coat. Tech. 206 (2011) 1120-1129.

[12] R. M. Mahamood, E. T. Akinlabi, Laser metal deposition of functionally graded Ti6Al4V/TiC, Mater. Des. 84 (2015) 402-410. 
[13] Z. M, Wang, E. O. Ezugwu, Titanium Alloys and Their Machinability a Review. J. Mater. Proc. Tech. 68 (1997) 262-270.

[14] E3-11, Standard Guide for Preparation of Metallographic Specimens, ASTM international Book of Standards, (2011) 03.01.

[15] R. M. Mahamood, E. T. Akinlabi, M. Shukla, S. Pityana. Scanning Velocity Influence on Microstructure, Microhardness and Wear Resistance Performance on Laser Deposited Ti6Al4V/TiC Composite. Mater. Des. 50 (2013) 656-666.

[16] ASTM E384 - 11e1, Standard Test Method for Knoop and Vickers Hardness of Materials, ASTM International Book of Standards, vol. 03.01, (2011).

[17] R. M. Mahamood, E. T. Akinlabi, M. Shukla, S. Pityana, Characterization of laser deposited Ti6A4V/TiC composite. Laser. Eng. 29(3-4) (2014) 197-213. 